\title{
Extreme points and descriptive sets
}

\author{
by
}

\author{
Robert P. Ka uf man (Urbana, Ill.)
}

\begin{abstract}
A class of closed, bounded, convex sets in the Banach space $c_{0}$ is shown to be a complete PCA set.
\end{abstract}

Introduction. Let $K$ denote a closed, bounded, convex set in a separable $B$-space $X$, and let ex $K$ denote its set of extreme points. It is possible that ex $K=\emptyset$, and also that ex $K$ fail to be a Borel set $([5,6])$. Hence it is natural to ask for the complexity of the set $C E$ of sets $K$ in $X$ having an extreme point. This question will be answered for $X=c_{0}$, after a digression on the class $F(M)$ of all closed subsets of a metric space $(M, d)$. This class can be quite mysterious $([4,7])$, but we mention only the rudiments. When $F(M)$ is provided with the Hausdorff metric - a minor adjustment is necessary when $d$ is unbounded-certain sets $[U]$ in $F(M)$ are open. Here $U$ is open in $M$ and

$$
A \in[U] \leftrightarrow A \cap U \neq \emptyset .
$$

When $d$ is totally bounded - equivalently, when $F(M)$ is separable - the sets $[U]$ generate the field of Borel sets, called the Effros Borel structure, and therefore the Borel structure in $F(M)$ has a definite meaning when $M$ is separable (since then there is some totally bounded metric). Some sets are always closed, for example the subset of $M \times F(M)$ defined by $m \in A$. When $X$ is a separable $B$-space, the convex sets form a $G_{\delta}$. To see this, let $\left(U_{n}\right)_{n=1}^{\infty}$ be a basis for the open sets; then $A$ is convex provided $A$ meets $\frac{1}{2}\left(U_{n}+U_{m}\right)$ whenever $A$ meets both $U_{n}$ and $U_{m}$. The Hausdorff metric in $F(X)$, relative to the usual metric, will be called the strong metric; that relative to a totally bounded metric in $X$ will be called a weak metric. (This has no relation to the weak topology.)

Let $E$ be the subset of $F(X) \times X \times X \times X$ containing elements $(A, x, y, z)$

\footnotetext{
Supported in part by the National Science Foundation
} 
such that

$$
x \in A, \quad y \in A, \quad z \in A, \quad x \neq y, \quad x \neq z, \quad x \in \overline{y z} .
$$

Then $E$ is a $G_{\delta}$ (for any weak metric in $F(X)$ ) and its projection on the first and second factors is the set of pairs $(A, x)$ such that $x \in A$ and $x$ is not an extreme point of $A$. From this we conclude that $C E$ is of class PCA (alias $\Sigma_{2}^{1}$ ) for a weak metric. Recent work on realization of PCA sets by means of sets in classical analysis is presented in $[1,2]$.

TheOREM. Let $\mathcal{S}$ be a PCA set in a compact metric space $M$. Then there is a mapping $m \rightarrow K(m)$ defined on $M$ such that

(i) $K(m)$ is a closed, bounded, convex subset of $c_{0}$.

(ii) $K(m)$ has an extreme point if and only if $m \in \mathcal{S}$.

(iii) The mapping is continuous from $M$ to the strong metric in $F\left(c_{0}\right)$.

Proof. This begins with some elementary topology and a summary of [6]. Being of class PCA, $\mathcal{S}$ is a continuous image $f\left(\mathcal{S}_{1}\right)$ of a certain $C A$ set $\mathcal{S}_{1}$ in a compact metric space $M_{1}$. By a device used in [6], matters can be so arranged that $f$ admits a continuous extension to all of $M_{1}$, mapping $M_{1}$ into $M$. Let $P\left(M_{1}\right)$ be the set of probability measures in $M_{1}$, with its $w^{*}$-topology, and $T$ an affine homeomorphism of $P\left(M_{1}\right)$ onto a compact set $C$ in $c_{0}$. Then there is a closed, bounded, convex set $K_{0}$ in $c_{0} \times c_{0}$ such that $([6])$

(i) $C \times\{0\}$ is contained in $K_{0}$.

(ii) The extreme points of $K_{0}$ are precisely the elements $\left(T\left(\delta_{m_{1}}\right), 0\right)$ with $m_{1} \in \mathcal{S}_{1}$.

Let $h$ be continuous on $M_{1}$ to $[0,1]$ and let $K(h)$ be the convex subset of $c_{0} \times c_{0} \times c_{0}$ containing all $(T(\mu), u, v)$ such that $(T(\mu), u) \in K_{0},\|v\| \leq \int h d \mu$. To determine ex $K(h)$, we recall that the unit ball of $c_{0}$ has no extreme points and therefore $(T(\mu), u, v)$ cannot be extreme if $\int h d \mu>0$. If, then, $(T(\mu), u, v)$ is extreme, then $v=0$, whence $(T(\mu), u)$ is extreme in $K_{0}$, and (as just observed) $\int h d \mu=0$. Conversely, suppose $(T(\mu), 0)$ is extreme and $\int h d \mu=0$; and suppose $(T(\mu), 0,0)=\frac{1}{2}\left(T\left(\mu_{1}\right), u_{1}, v_{1}\right)+\frac{1}{2}\left(T\left(\mu_{2}\right), u_{2}, v_{2}\right)$. Then $\mu_{1}=\mu_{2}=\mu, u_{1}=u_{2}=0$, and consequently $v_{1}=v_{2}=0$. Thus, in summary

$K(h)$ has an extreme point $\Leftrightarrow h$ has a zero in $\mathcal{S}_{1}$.

Moreover, the Hausdorff distance between $K\left(h_{1}\right)$ and $K\left(h_{2}\right)$ is at most $\left\|h_{1}-h_{2}\right\|$.

Let $\varrho$ be a metric in $M$ and suppose $\varrho \leq 1$, and let $h\left(m, m_{1}\right)=$ $\varrho\left(m, f\left(m_{1}\right)\right)$. Then $h(m, \cdot)$ is continuous on $M_{1}$, and $h(m, \cdot)$ has a zero in $\mathcal{S}_{1}$ $\Leftrightarrow m \in f\left(\mathcal{S}_{1}\right)=\mathcal{S}$. Using these functions for $h$ in $K(h)$ we obtain the theorem. 
Conclusion. We mention some problems, of uncertain difficulty, related to the main result; background material is presented in [3].

(i) Find other spaces $X$ in place of $c_{0}$. Since $l^{1}$ has RNP, the most likely candidate is $L^{1}$. Besides this, there are the separable subspaces of the non-RNP spaces of Stegall ([3], Ch. 4).

(ii) What happens when extreme points are replaced by denting points, exposed points, strongly exposed points, etc. ([3], Ch. 3)?

(iii) Classify the sets $K$ such that $K=\overline{\mathrm{co}}(\operatorname{ex} K)$.

(iv) Fixing $K$, classify the set of points represented by an integral over ex $K$ ([3], Ch. 6).

\section{References}

[1] H. Becker, Pointwise limits of subsequences and $\Sigma_{2}^{1}$ sets, Fund. Math. 128 (1987), 159-170.

[2] H. Becker, S. Kahane and A. Louveau, Some complete $\Sigma_{2}^{1}$ sets in harmonic analysis, preprint, Univ. Paris VI, 1991.

[3] D. G. Bourgin, Geometric Aspects of Convex Sets with the Radon-Nikodym Property, Lecture Notes in Math. 993, Springer, 1983.

[4] E. G. Effros, Convergence of closed subsets in a topological space, Proc. Amer. Math. Soc. 16 (1965), 929-931.

[5] J. E. Jayne and C. A. Rogers, The extremal structure of convex sets, J. Funct. Anal. 26 (1977), 251-288.

[6] R. Kaufman, Co-analytic sets and extreme points, Bull. London Math. Soc. 19 (1987), $72-74$.

[7] J. Saint-Raymond, La structure Borélienne d'Effros est-elle standard?, Fund. Math. 50 (1978), 201-210.

DEPARTMENT OF MATHEMATICS

UNIVERSITY OF ILLINOIS AT URBANA-CHAMPAIGN

1409 WEST GREEN STREET

URBANA, ILLINOIS 61801

U.S.A. 